\title{
Delta-24 adenoviral therapy for glioblastoma: evolution from the bench to bedside and future considerations
}

\author{
Chibawanye I. Ene, MD, PhD, ${ }^{1}$ Juan Fueyo, MD, ${ }^{1,2}$ and Frederick F. Lang, MD' \\ Departments of ${ }^{1}$ Neurosurgery and ${ }^{2}$ Neuro-Oncology, The University of Texas MD Anderson Cancer Center, Houston, Texas
}

\begin{abstract}
Delta-24-based oncolytic viruses are conditional replication adenoviruses developed to selectively infect and replicate in retinoblastoma $1(\mathrm{Rb})$-deficient cancer cells but not normal cell with intact $\mathrm{Rb} 1$ pathways. Over the years, there has been a significant evolution in the design of Delta-24 based on a better understanding of the underlying basis for infection, replication, and spread within cancer. One example is the development of Delta-24-RGD (DNX-2401), where the arginine-glycine-aspartate (RGD) domain enhances the infectivity of Delta-24 for cancer cells. DNX-2401 demonstrated objective biological and clinical responses during a phase I window of opportunity clinical trial for recurrent human glioblastoma. In long-term responders (> 3 years), there was evidence of immune infiltration ( $T$ cells and macrophages) into the tumor microenvironment with minimal toxicity. Although more in-depth analysis and phase III studies are pending, these results indicate that Delta-24-based adenovirus therapy may induce an antitumor response in glioblastoma, resulting in long-term antitumor immune response. In this review, the authors discuss the preclinical and clinical development of Delta-24 oncolytic adenoviral therapy for glioblastoma and describe structural improvements to Delta-24 that have enhanced its efficacy in vivo. They also highlight ongoing research that attempts to address the remaining obstacles limiting efficacy of Delta-24 adenovirus therapy for glioblastoma.

https://thejns.org/doi/abs/10.3171/2020.11.FOCUS20853
\end{abstract}

KEYWORDS adenovirus; Delta-24; oncolytic virus; retinoblastoma gene mutation; glioblastoma

$\mathrm{T}$ HE concept of using viruses to target cancer has been clinically tested since the $1950 \mathrm{~s} .{ }^{1,2}$ Since then, multiple oncolytic viruses have been developed based on normal viruses that target human cells, including herpes simplex viruses (HSVs), adenoviruses, reovirus, vaccinia virus, measles virus, and polioviruses. ${ }^{3} \mathrm{Al}-$ though these viruses have different genomic backbones, they share similar principles for cancer therapy: selective infection of cancer cells and some replicate only within cancer cells but not normal cells. Replication results in tumor cell lysis and the release of tumor and viral antigens that activate the host immune system to seek and eliminate cancer-specific antigen- or viral antigen-expressing cancer cells. ${ }^{2}$

In 2015, the FDA approved talimogene laherparepvec (T-VEC), a genetically modified HSV-based oncolytic virus for advanced melanoma, the first of such approvals for an oncolytic virus in oncology. ${ }^{4}$ In glioblastoma, there have been extensive preclinical and clinical studies evaluating multiple varieties of oncolytic virus therapies, including poliovirus-, HSV-, and adenovirus-based therapies, such as Delta-24, with promising evidence of efficacy. ${ }^{5-7}$ To date, however, no oncolytic virus has been approved for use in patients with glioblastoma. Results from a phase I clinical trial evaluating Delta-24 in recurrent glioblastoma provide optimism that this class of oncolytic adenoviruses may soon gain FDA approval for glioblastoma. ${ }^{7}$ Here, we summarize the mechanisms underlying Delta-24 activity in human glioblastoma based on preclinical and clinical studies. We also discuss the obstacles impeding progress of Delta-24 in glioblastoma and highlight research that attempts to address these obstacles.

\section{Retinoblastoma Pathway Dysfunction Mediates Selectivity of Delta-24 for Cancer Cells}

Adenoviruses are double-stranded DNA viruses with a broad range of hosts, including humans. ${ }^{2}$ In humans, adenoviruses have been linked to respiratory diseases, conjunctivitis, and gastroenteritis. ${ }^{8}$ Hence, there is a wealth of knowledge about the biology of adenovirus in humans, making it easier to manipulate and tailor for cancer.

One unique advantage of adenoviruses that makes them strong candidates for cancer therapy is their ability to target specific cell cycle regulators. ${ }^{8}$ Many cancers, including

ABBREVIATIONS DNX-2401 = Delta-24-RGD, second generation; $\mathrm{HSV}=$ herpes simplex virus; $\mathrm{ICI}=$ immune checkpoint inhibitor; $m E 1 \mathrm{~A}=$ mutant $\mathrm{E} 1 \mathrm{~A} ; \mathrm{Rb}=$ retinoblastoma; $\mathrm{RGD}$ = arginine-glycine-aspartate; $\mathrm{TMZ}=$ temozolomide. 

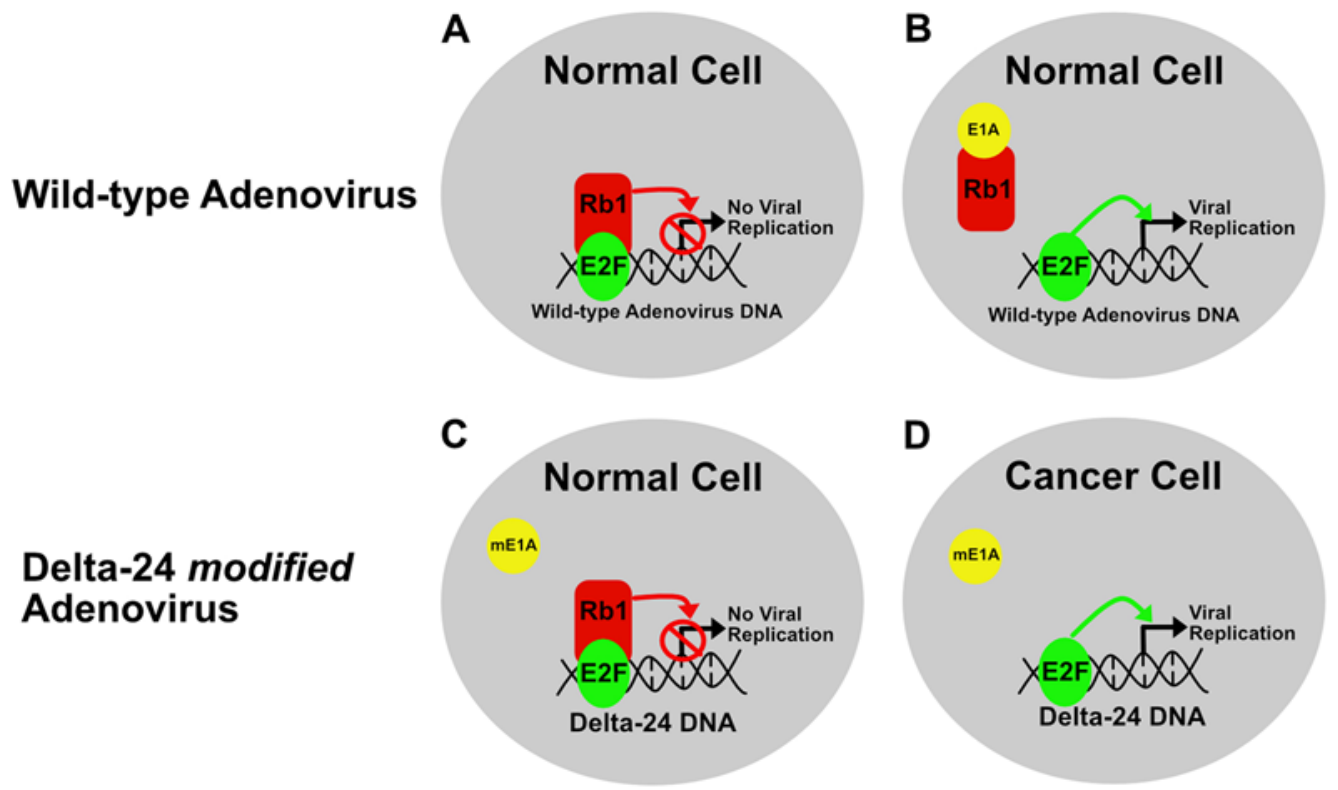

\begin{abstract}
FIG. 1. Selectivity of Delta-24 oncolytic viruses for tumor cells with Rb pathway aberration. A and B: In normal cells, Rb1 inhibits cell cycle progression (A). On wild-type adenovirus infection, virus protein E1A binds Rb1, thereby inhibiting its ability to repress $\mathrm{E} 2 \mathrm{~F}$. In the absence of Rb1, E2F enhances transcription of genes involved in viral replication; therefore, the wild-type adenovirus replicates within the cell. $\mathbf{C}$ and $\mathrm{D}$ : Delta-24 adenovirus is a mutant form of wild-type adenovirus with deletion of the Rb1-binding domain of E1A (mutant E1A [mE1A]). After Delta-24 treatment, the virus cannot replicate in normal cells since mE1A cannot bind and inhibit the suppressive effects of Rb1 on the cell cycle and viral replication (C). Delta-24, however, is able to replicate in Rb1deficient cancer cells since E2F (uninhibited) drives transcriptional activity of viral replication genes (D).
\end{abstract}

glioblastoma, have defective retinoblastoma $(\mathrm{Rb})$ tumor suppressor pathways. ${ }^{9}$ The Delta-24 adenovirus is based on human adenovirus type 5 and has a 24-base pair deletion in the Rb-binding domain of the ElA gene (mutant E1A $[\mathrm{mE} 1 \mathrm{~A}]) .{ }^{10} \mathrm{E} 1 \mathrm{~A}$ encodes a protein that binds Rb-protein, releasing E2F, which serves as a transcription factor for genes that promote progression into the S-phase of the cell cycle, which enhances viral replication (Fig. 1). Therefore, Delta-24 cannot replicate in normal cells since mE1A cannot bind $\mathrm{Rb}$ protein to release $\mathrm{E} 2 \mathrm{~F}$. In cancer cells, however, the $\mathrm{Rb}$ deficiency indicates that there is $\mathrm{E} 2 \mathrm{~F}$ protein in the free state that drives cells into the S-state; therefore, viral replication is enhanced. This biology has been the backbone of the Delta-24 adenoviral-based oncolytic virus therapy and mediates selectivity of Delta-24 for cancer cells.

The steps for oncolytic virus infection to antitumor immune response, however, is plagued by normal attempts by the host immune response to eradicate the viral infection, which may impact the effectiveness of the virus and consequently affect the antitumor immune response. ${ }^{11} \mathrm{Be}-$ low, we discuss how lessons from preclinical and clinical studies have resulted in the evolution and improvements to the original Delta-24 virus, with significantly improved infectivity and an ability to elicit an antitumor immune response in glioblastoma.

\section{Structural Modifications to Delta-24 Enhance Infectivity and Antitumor Immune Response}

Over the last few decades, there have been modifications to Delta-24 that improve its infectivity, replication, or abil- ity to induce or sustain an antitumor response. The human adenovirus 5 (from which the Delta-24 vector is based) binds to coxsackievirus and adenovirus receptor (CAR) on the cell surface prior to entry into host cells. ${ }^{12}$ Some cancers, including glioblastoma, however, express low levels of CAR, resulting in poor infectivity of glioblastoma cells by Delta-24 (first generation). ${ }^{13}$ To overcome this obstacle, a gene that encodes an arginine-glycine-aspartate (RGD) peptide into the fiber knob receptor of the virus was introduced into Delta-24 (termed Delta-24-RGD or DNX-2401, second generation). ${ }^{14}$ The RGD peptide has strong affinity for integrins, which are overexpressed on cancer cells. This RGD modification significantly improved the infection rate of DNX-2401 for cancer cells, including glioblastoma. ${ }^{13,15}$ Results of a phase I clinical trial for DNX-2401 (second generation) in recurrent glioblastoma have been reported ${ }^{7}$ and are discussed in the section below.

In addition to the oncolytic effects of Delta-24-RGD, there was evidence of an antitumor immune response in a subset of patients (long-term survivors) during clinical trials for glioblastoma. Therefore, to further enhance the antitumor immune response elicited by Delta-24-RGD, a new generation of Delta-24-RGD oncolytic viruses armed immune activators, were designed. Delta-24-RGDOX (third generation, DNX-2440) is armed with genetic information encoding mouse OX40 L, the ligand for the Tcell-activating receptor OX40 on the surface of T cells (Delta-24-RGDOX). ${ }^{16}$ Intratumoral injection of Delta24-RGDOX resulted in more T-cell infiltration and better survival of immunocompetent mice relative to DNX- 
TABLE 1. Human clinical trials evaluating the safety and efficacy of DNX-2401

\begin{tabular}{|c|c|c|c|c|c|c|}
\hline Clinical Trial & Title & Phase & Tumor & Interventions & Country & Status \\
\hline NCT0219716946 & $\begin{array}{l}\text { DNX-2401 With Interferon Gamma (IFN- } \gamma \text { ) for } \\
\text { Recurrent Glioblastoma or Gliosarcoma } \\
\text { Brain Tumors (TARGET-I) }\end{array}$ & I & $\begin{array}{l}\text { Recurrent } \\
\text { glioblastoma }\end{array}$ & $\begin{array}{l}\text { Arm A: DNX-2401 alone } \\
\text { Arm B: DNX-2401 + IFN- } \gamma\end{array}$ & US & Completed \\
\hline NCT00805376 & $\begin{array}{l}\text { DNX-2401 (Formerly Known as Delta-24-RGD- } \\
\text { 4C) for Recurrent Malignant Gliomas }\end{array}$ & 1 & $\begin{array}{l}\text { Recurrent } \\
\text { glioblastoma }\end{array}$ & $\begin{array}{c}\text { Arm A: DNX-2401 alone } \\
\text { Arm B: DNX-2401 + surgery }\end{array}$ & US & Completed \\
\hline NCT0195673447 & $\begin{array}{l}\text { Virus DNX2401 and Temozolomide in Recur- } \\
\text { rent Glioblastoma (D24GBM) }\end{array}$ & I & $\begin{array}{c}\text { Recurrent } \\
\text { glioblastoma }\end{array}$ & DNX2401 \& TMZ & Spain & Completed \\
\hline NCT0158251648 & $\begin{array}{l}\text { Safety Study of Replication-competent } \\
\text { Adenovirus (Delta-24-rgd) in Patients with } \\
\text { Recurrent Glioblastoma }\end{array}$ & I & $\begin{array}{l}\text { Recurrent } \\
\text { glioblastoma }\end{array}$ & Intratumoral Delta-24 RGD & $\begin{array}{l}\text { The } \\
\text { Netherlands }\end{array}$ & Completed \\
\hline NCT0317803249 & $\begin{array}{l}\text { Oncolytic Adenovirus, DNX-2401, for Naive Dif- } \\
\text { fuse Intrinsic Pontine Gliomas }\end{array}$ & I & DIPG & $\begin{array}{l}\text { Brain infusion of the virus } \\
\text { through the cerebellar peduncle }\end{array}$ & Spain & $\begin{array}{l}\text { Active, not } \\
\text { recruiting }\end{array}$ \\
\hline NCT0279840650 & $\begin{array}{l}\text { Combination Adenovirus + Pembrolizumab to } \\
\text { Trigger Immune Virus Effects (CAPTIVE) }\end{array}$ & I & $\begin{array}{l}\text { Recurrent } \\
\text { glioblastoma }\end{array}$ & DNX-2401 + pembrolizumab & $\begin{array}{l}\text { US \& } \\
\text { Canada }\end{array}$ & $\begin{array}{l}\text { Active, not } \\
\text { recruiting }\end{array}$ \\
\hline NCT0389656851 & $\begin{array}{l}\text { Allogeneic Bone Marrow Human Mesenchymal } \\
\text { Stem Cells Loaded With A Tumor Selective } \\
\text { Oncolytic Adenovirus (DNX-2401) }\end{array}$ & I & $\begin{array}{l}\text { Recurrent } \\
\text { glioblastoma }\end{array}$ & $\begin{array}{c}\text { Arm A: DNX-2401 alone } \\
\text { Arm B: DNX-2401 + surgery }\end{array}$ & US & Recruiting \\
\hline
\end{tabular}

DIPG = diffuse intrinsic pontine glioma.

2401. Delta-24-RGDOX is still being evaluated in a phase I clinical trial for recurrent glioblastoma (identifier no. NCT03714334, clinicaltrials.gov).

Another design incorporates a costimulatory ligand GITRL (Delta-24-GREAT [glucocorticoid receptor enhanced activity of $\mathrm{T}$ cells]) (fourth generation).$^{17}$ In preclinical studies, Delta-24-GREAT induced a robust antiviral and antiglioma immune response with increased frequency of central memory CD8+ T cells. Furthermore, rechallenging surviving mice with a second implantation of glioma cells did not result in tumor growth, but implantation of melanoma cells resulted in diminished survival, indicating a glioma-specific immune response following Delta-24-GREAT treatment. Human clinical trials evaluating Delta-24-GREAT in glioblastoma are pending.

\section{Clinical Trials Evaluating DNX-2401 for Glioblastoma \\ DNX-2401 for Recurrent Glioblastoma}

Following extensive preclinical studies, Lang et al. reported results from a phase I, dose escalation biological endpoint, or window of opportunity, clinical trial in 37 patients with recurrent glioblastoma (Table 1). ${ }^{7}$ Here, following a biopsy confirming recurrent tumor, patients received intratumoral injections of DNX-2401 as intervention. The study was designed to assess safety $(\operatorname{arm} \mathrm{A}, \mathrm{n}=$ 25 ) and mechanism of action ( $\operatorname{arm} B, n=12)$. In $\operatorname{arm} A, a$ dose escalation was performed over 8 doses to determine safety and response. Patients in arm B underwent intratumoral injection of DNX-2401 through an implanted catheter, followed 14 days later by en bloc resection to acquire a posttreatment specimen. Results from arm A showed that $20 \%$ of patients survived $>3$ years from treatment and at least 3 patients showing a $>95 \%$ reduction in enhancing tumor resulting in $>3$ years of progression-free survival. Evaluation of posttreatment specimens from arm
B demonstrated that DNX-2401 replicates and spreads within the tumor, indicating direct virus-induced oncolysis in patients. There was also radiographic evidence of inflammation, and histological evaluation demonstrated infiltration by $\mathrm{CD} 8+\mathrm{T}$ cells. These results indicate that DNX-2401 induction of long-term survivors was likely due to both its direct oncolytic effects and elicitation of immune-mediated antiglioma response. The underlying mechanism mediating susceptibility to DNX-2401 in the long-term survivors remains an intense area of research (discussed in subsequent sections). Given this promising phase I result, however, phase II clinical trials are planned for DNX-2401 in recurrent glioblastoma.

\section{DNX-2401 for Pediatric High-Grade Gliomas}

The indications for DNX-2401 may go beyond glioblastoma given the ability for DNX-2401 to selectively target tumor cells with abnormal Rb1 pathways. Preclinical studies of DNX-2401 in pediatric high-grade gliomas and diffuse intrinsic pontine gliomas in mouse models demonstrated efficacy across multiple subtypes of pediatric mouse and human glioma cell lines in vitro and in vivo. ${ }^{18}$ Given these preclinical findings, a phase I clinical trial of DNX-2401 for newly diagnosed diffuse intrinsic pontine glioma was initiated and just completed patient accrual (clinical trial no. NCT03178032). Results from this study will hopefully provide insights into the utility of DNX-2401 for other lethal CNS tumors such as pediatric gliomas, which are molecularly distinct from adult high-grade gliomas.

\section{DNX-2401 Administration Via Mesenchymal Stem Cells}

Following treatment with oncolytic viruses, an antiviral immune response (natural killer cells and macrophages) mediates early clearance of the virus. ${ }^{19,20}$ Although this represents a normal response to viral infection, this antiviral response may mitigate the oncolytic effects of the 
virus within tumors since the virus has limited time for replication within tumor cells. Given this response, several approaches have been proposed that would protect the virus from detection by the immune system, including packaging within stem cells. ${ }^{21-23}$ Stem cells have demonstrated the ability to home to tumors and therefore may serve as delivery vehicles for DNX-2401 to tumors even within the CNS. Mesenchymal stem cells and neural stem cells carrying adenovirus have demonstrated antiglioma activity in vivo. ${ }^{24-28}$ There is an ongoing phase I clinical trial evaluating systemic mesenchymal stem cell-based delivery of DNX-2401 for patients with recurrent glioblastoma (identification no. NCT03896568). Alternatively, mesenchymal stem cells embedded in hydrogels directly placed in the tumor bed provide another avenue that could prevent the systemic toxicity or clearance associated with intraarterial delivery. ${ }^{29}$ This approach remains in the preclinical stage of development.

\section{DNX-2401 With Chemotherapy}

The combination of DNX-2401 with various forms of chemotherapy such as temozolomide (TMZ) have shown efficacy in several preclinical models of glioblastoma. ${ }^{30}$ Mechanistically, it is conceivable that DNX-2401 and chemotherapy could synergistically result in tumor regression. In one study, the administration of DNX-2401 was shown to decrease the $\mathrm{IC}_{50}$ of $\mathrm{TMZ}$ in vitro. ${ }^{30}$ It is likely that both agents are working via independent pathways to suppress tumor growth and their effects are additive. It is also plausible, however, that, in addition to direct effects on MGMT-methylated tumor cells, TMZ-based chemotherapy also suppresses an antiviral immune response allowing DNX-2401 to induce oncolysis. This is based on a preclinical study evaluating the effect of TMZ administration before DNX-2401 treatment or after DNX-2401 treatment in immunocompetent mice. ${ }^{31}$ Both TMZ treatment before and after DNX-2401 treatment significantly improved survival relative to controls and TMZ alone. Only TMZ treatment after DNX-2401, however, significantly improved survival relative to DNX-2401 treatment alone. Tissue analysis showed that the TMZ before DNX-2401 regimen led to reduced dendritic cell and CD8+ cell infiltration, unlike the TMZ after DNX-2401 regimen, which showed significantly higher CD8+ infiltration associated with tumor-specific recognition. These findings indicate that administration of TMZ before DNX-2401 may suppress both antiviral and antitumor immune response. Conversely, TMZ given after DNX-2401 treatment may blunt antiviral responses but not antitumor responses resulting in enhanced T-cell infiltration and survival. The mechanism mediating an immune response against DNX-2401 and whether this antiviral immune response is distinct from the antitumor immune response remain unknown. This area of research warrants further investigation since strategies that suppress an antiviral immune response without affecting an antitumor immune response could boost the efficacy of oncolytic viruses such as DNX-2401. The combination of DNX-2401 with TMZ is currently being evaluated in human clinical trials (identifier no. NCT10956734).

\section{DNX-2401 With Immune Checkpoint Inhibitors}

Unlike other cancers such as melanoma, monotherapy with immune checkpoint inhibitors (ICIs) have shown mixed results for glioblastoma. ${ }^{31-34}$ While most trials for glioblastoma have been negative, some trials assessing checkpoint inhibitors administered in the neoadjuvant setting have shown efficacy. ${ }^{33}$ This indicates that ICIs may work for glioblastoma under specific conditions such as patient-specific tumor genomic alterations, timing of ICI administration, and baseline tumor immune microenvironment composition. ${ }^{33,35}$ One difference between glioblastoma and other ICI-susceptible cancers is that glioblastoma has a cold or noninflammatory tumor immune microenvironment. ${ }^{36}$ The administration of oncolytic viruses such as DNX-2401 could modify the tumor microenvironment toward a hot or inflammatory phenotype by recruiting immune cells into the tumor. This indicates that oncolytic viruses such as DNX-2401 may serve as a primer for enhancing the efficacy of ICIs in glioblastoma. ${ }^{7,13}$ To this end, there is an ongoing clinical trial evaluating the combination of DNX-2401 and anti-PD1 antibody for glioblastoma (identifier no. NCT02798406).

\section{Current Challenges and Future Considerations for DNX-2401 in Glioblastoma Lack of Representative Preclinical Models}

Several animal models have been used to test the preclinical safety and efficacy of oncolytic adenoviruses such as DNX-2401. 13,17,18,31,37 Most preclinical models for glioma exist in mice. Unfortunately, the replication of Delta-24 in mouse cells is less efficient relative to human cell types. ${ }^{38}$ Therefore, multiple injections are typically required to achieve high viral titers within mouse tumors in vivo. As an alternative, human patient-derived glioma xenografts have been used to provide insights into mechanisms of viral replication and oncolysis within human cells in vivo. ${ }^{13}$ Since human xenografts are propagated in immunocompromised mice, however, this setting may not be an ideal environment for fully characterizing the complex cascade of immune cell infiltration following Delta-24 administration. The Syrian hamster brain tumor model not only provides an immunocompetent background but also a species that allows for viral replication similar to that in humans. ${ }^{39}$ Therefore, hamsters provide an alternative to mouse models for characterizing the immune response to Delta- 24 in an immunocompetent setting. Unlike the mouse model, where there have been numerous studies with a vast array of validated reagents, the hamster model is hampered by limited glioma cell lines and a lack of hamster-specific reagents, including antibodies. ${ }^{40}$ Given similarities to human cell lines, the hamster glioma models and reagents warrant more validation, as they provide a preclinical environment that recapitulates the human microenvironment following Delta-24 administration.

Another emerging aspect of preclinical study that allows for studying both oncolytic effects and antitumor immune response in a human background are cerebral organoids. ${ }^{41}$ Here, human stem cells are used to generate multiple cell types, including immune cells, within the organoid. Characterization of these cerebral organoids have 
demonstrated complex microenvironments that include both extracellular matrix and immune cells. ${ }^{42}$ On addition of patient-specific glioma cells to the organoids, there is evidence of interaction between tumor cells and organoid microenvironment that recapitulates the patient-specific tumor microenvironment. Over time, we anticipate that more studies will utilize this model for characterizing response of patient-specific organoids to different treatments, including oncolytic virus such as Delta-24 in a complex human microenvironment in vitro.

\section{Balancing Antiviral Immune Response, Oncolysis, and Antitumor Response}

Another aspect of DNX-2401 treatment that limits efficacy is an antiviral response driven predominantly by the innate immune system. ${ }^{19,43}$ Within minutes of virus administration, preclinical models have shown that components of the innate immune system, including macrophages and natural killer cells, are activated and they mediate an antiviral immune response. ${ }^{19,20}$ This process results in a drop in viral titers and limits infection of tumor cells and oncolysis and, consequently, the antitumor immune response that follows. Furthermore, the mechanisms mediating an antitumor immune response may also contribute to an antiviral immune response, which further decreases viral titers, including interferon gamma (IFN- $\gamma$ ). To date, it remains unclear if we can selectively limit the antiviral response without impeding the antitumor immune response. One way to mitigate the antiviral response is delivery of DNX-2401 within stem cells. ${ }^{24,25,29}$ This process helps shield the virus from detection by the innate immune system and allows spreading and tumor infection.

The efficacy of oncolytic virus has been shown to depend on both oncolysis and the antitumor immune response. ${ }^{44}$ The relevance of the balance between oncolysis and antitumor response for clinical efficacy remains controversial and may be tumor specific. Preclinical data indicate that some tumors may be more susceptible to oncolysis, and therefore this aspect dictates clinical outcomes, while other models show that oncolysis is only a minimal part of the response as the antitumor component is what ultimately dictates clinical response. ${ }^{45}$ This indicates that intrinsic factors such as tumor-specific mutations may influence susceptibility to DNX-2401. Several genomic factor such as Rb pathway dysfunction (INK4A/ARF deletion, $C D K 4 / 6$ amplification) have been shown to enhance viral replication and oncolysis. ${ }^{13}$ The relevance of mutations for DNX-2401-induced oncolysis in glioblastoma, however, remains understudied. We anticipate that ongoing human clinical trials evaluating DNX-2401 for glioblastoma will hopefully uncover patient-specific factors that influence susceptibility to oncolysis and antitumor immune response.

\section{Conclusions}

There are currently no FDA-approved oncolytic virus therapies for glioblastoma. DNX-2401 is the most studied adenovirus-based vector therapy in glioblastoma, and, given promising phase I and II trial results, it may become the first FDA-approved viral treatment for glioblastoma.? Although there are several obstacles impeding the efficacy of DNX-2401, some patients show dramatic response to DNX-2401. It is our hope that large-scale immune microenvironment studies that characterize the complex interactions between immune cells and virus will hopefully allow for the discovery of patient-specific conditions such as underlying tumor mutations, HLA (human leukocyte antigen) subtype, immune microenvironment composition, or preexisting neutralizing antibodies that predict susceptibility and resistance to treatment.

\section{Acknowledgments}

This study was supported by the National Cancer Institute (1R01CA214749, 1R01CA247970, and 2P50CA127001), The University of Texas MD Anderson Moon Shots Program, The Broach Foundation for Brain Cancer Research, The Elias Family Fund, The Priscilla and Jason Hiley Fund, The Baumann Family/CureFest Fund, The Jim and Pam Harris Fund, The Gene Pennebaker Brain Cancer Fund, The Schneider Memorial Fund, The Sweet Family Cancer Research Fund, The Dr. Marnie Rose Foundation, The Gold Family Memorial Fund, and The Sorenson Foundation (all to F.F.L.)

\section{References}

1. Larson C, Oronsky B, Scicinski J, et al. Going viral: a review of replication-selective oncolytic adenoviruses. Oncotarget. 2015;6(24):19976-19989.

2. Kiyokawa J, Wakimoto H. Preclinical and clinical development of oncolytic adenovirus for the treatment of malignant glioma. Oncolytic Virother. 2019;8:27-37.

3. Alemany R. Viruses in cancer treatment. Clin Transl Oncol. 2013;15(3):182-188.

4. Andtbacka RH, Kaufman HL, Collichio F, et al. Talimogene laherparepvec improves durable response rate in patients with advanced melanoma. J Clin Oncol. 2015;33(25):2780-2788.

5. Desjardins A, Gromeier M, Herndon JE II, et al. Recurrent glioblastoma treated with recombinant poliovirus. $N$ Engl J Med. 2018;379(2):150-161.

6. Grandi P, Peruzzi P, Reinhart B, et al. Design and application of oncolytic HSV vectors for glioblastoma therapy. Expert Rev Neurother. 2009;9(4):505-517.

7. Lang FF, Conrad C, Gomez-Manzano C, et al. Phase I study of DNX-2401 (Delta-24-RGD) oncolytic adenovirus: replication and immunotherapeutic effects in recurrent malignant glioma. J Clin Oncol. 2018;36(14):1419-1427.

8. Chu RL, Post DE, Khuri FR, Van Meir EG. Use of replicating oncolytic adenoviruses in combination therapy for cancer. Clin Cancer Res. 2004;10(16):5299-5312.

9. Brennan CW, Verhaak RG, McKenna A, et al. The somatic genomic landscape of glioblastoma. Cell. 2013;155(2):462-477.

10. Fueyo J, Gomez-Manzano C, Alemany R, et al. A mutant oncolytic adenovirus targeting the $\mathrm{Rb}$ pathway produces anti-glioma effect in vivo. Oncogene. 2000;19(1):2-12.

11. Lemos de Matos A, Franco LS, McFadden G. Oncolytic viruses and the immune system: the dynamic duo. Mol Ther Methods Clin Dev. 2020;17:349-358.

12. Bergelson JM, Cunningham JA, Droguett G, et al. Isolation of a common receptor for Coxsackie B viruses and adenoviruses 2 and 5. Science. 1997;275(5304):1320-1323.

13. Fueyo J, Alemany R, Gomez-Manzano C, et al. Preclinical characterization of the antiglioma activity of a tropismenhanced adenovirus targeted to the retinoblastoma pathway. J Natl Cancer Inst. 2003;95(9):652-660.

14. Wickham TJ, Mathias P, Cheresh DA, Nemerow GR. Integrins alpha $v$ beta 3 and alpha $v$ beta 5 promote adenovirus internalization but not virus attachment. Cell. 1993;73(2):309-319.

15. Jiang H, Gomez-Manzano C, Aoki H, et al. Examination of 
the therapeutic potential of Delta-24-RGD in brain tumor stem cells: role of autophagic cell death. J Natl Cancer Inst. 2007;99(18):1410-1414.

16. Jiang H, Rivera-Molina Y, Gomez-Manzano C, et al. Oncolytic adenovirus and tumor-targeting immune modulatory therapy improve autologous cancer vaccination. Cancer Res. 2017;77(14):3894-3907.

17. Rivera-Molina Y, Jiang H, Fueyo J, et al. GITRL-armed Delta-24-RGD oncolytic adenovirus prolongs survival and induces anti-glioma immune memory. Neurooncol Adv. 2019; 1(1):vdz009.

18. Martínez-Vélez N, Garcia-Moure M, Marigil M, et al. The oncolytic virus Delta-24-RGD elicits an antitumor effect in pediatric glioma and DIPG mouse models. Nat Commun. 2019;10(1):2235.

19. Alvarez-Breckenridge CA, Yu J, Price R, et al. NK cells impede glioblastoma virotherapy through NKp30 and NKp46 natural cytotoxicity receptors. Nat Med. 2012;18(12):1827-1834.

20. Fulci G, Dmitrieva N, Gianni D, et al. Depletion of peripheral macrophages and brain microglia increases brain tumor titers of oncolytic viruses. Cancer Res. 2007;67(19):9398-9406.

21. Aboody KS, Brown A, Rainov NG, et al. Neural stem cells display extensive tropism for pathology in adult brain: evidence from intracranial gliomas. Proc Natl Acad Sci U S A. 2000;97(23):12846-12851.

22. Lesniak MS. Targeted therapy for malignant glioma: neural stem cells. Expert Rev Neurother. 2006;6(1):1-3.

23. Martinez-Quintanilla J, Seah I, Chua M, Shah K. Oncolytic viruses: overcoming translational challenges. J Clin Invest. 2019;129(4):1407-1418

24. Yong RL, Shinojima N, Fueyo J, et al. Human bone marrowderived mesenchymal stem cells for intravascular delivery of oncolytic adenovirus Delta24-RGD to human gliomas. Cancer Res. 2009;69(23):8932-8940.

25. Sonabend AM, Ulasov IV, Tyler MA, et al. Mesenchymal stem cells effectively deliver an oncolytic adenovirus to intracranial glioma. Stem Cells. 2008;26(3):831-841.

26. Ahmed AU, Thaci B, Tobias AL, et al. A preclinical evaluation of neural stem cell-based cell carrier for targeted antiglioma oncolytic virotherapy. J Natl Cancer Inst. 2013;105(13):968-977.

27. Tyler MA, Ulasov IV, Sonabend AM, et al. Neural stem cells target intracranial glioma to deliver an oncolytic adenovirus in vivo. Gene Ther. 2009;16(2):262-278.

28. Kim CK, Ahmed AU, Auffinger B, et al. N-acetylcysteine amide augments the therapeutic effect of neural stem cell-based antiglioma oncolytic virotherapy. Mol Ther. 2013;21(11):2063-2073.

29. Parker Kerrigan BC, Shimizu Y, Andreeff M, Lang FF. Mesenchymal stromal cells for the delivery of oncolytic viruses in gliomas. Cytotherapy. 2017;19(4):445-457.

30. Alonso MM, Gomez-Manzano C, Bekele BN, et al. Adenovirus-based strategies overcome temozolomide resistance by silencing the O6-methylguanine-DNA methyltransferase promoter. Cancer Res. 2007;67(24):11499-11504.

31. Kleijn A, van den Bossche W, Haefner ES, et al. The sequence of Delta24-RGD and TMZ administration in malignant glioma affects the role of CD8 $+\mathrm{T}$ cell anti-tumor activity. Mol Ther Oncolytics. 2017;5:11-19.

32. Larkin J, Chiarion-Sileni V, Gonzalez R, et al. Five-year survival with combined nivolumab and ipilimumab in advanced melanoma. N Engl J Med. 2019;381(16):1535-1546.

33. Cloughesy TF, Mochizuki AY, Orpilla JR, et al. Neoadjuvant anti-PD-1 immunotherapy promotes a survival benefit with intratumoral and systemic immune responses in recurrent glioblastoma. Nat Med. 2019;25(3):477-486.

34. Reardon DA, Brandes AA, Omuro A, et al. Effect of nivolumab vs bevacizumab in patients with recurrent glioblastoma: the CheckMate 143 phase 3 randomized clinical trial. JAMA Oncol. 2020;6(7):1003-1010.

35. Parsa AT, Waldron JS, Panner A, et al. Loss of tumor sup- pressor PTEN function increases B7-H1 expression and immunoresistance in glioma. Nat Med. 2007;13(1):84-88.

36. Duan Q, Zhang H, Zheng J, Zhang L. Turning cold into hot: firing up the tumor microenvironment. Trends Cancer. 2020; 6(7):605-618.

37. Huse JT, Holland EC. Genetically engineered mouse models of brain cancer and the promise of preclinical testing. Brain Pathol. 2009;19(1):132-143.

38. Zhang L, Hedjran F, Larson C, et al. A novel immunocompetent murine model for replicating oncolytic adenoviral therapy. Cancer Gene Ther. 2015;22(1):17-22.

39. Sonabend AM, Ulasov IV, Han Y, et al. Biodistribution of an oncolytic adenovirus after intracranial injection in permissive animals: a comparative study of Syrian hamsters and cotton rats. Cancer Gene Ther. 2009;16(4):362-372.

40. Jiang H, Clise-Dwyer K, Ruisaard KE, et al. Delta-24-RGD oncolytic adenovirus elicits anti-glioma immunity in an immunocompetent mouse model. PLoS One. 2014;9(5):e97407.

41. Linkous A, Balamatsias D, Snuderl M, et al. Modeling patient-derived glioblastoma with cerebral organoids. Cell Rep. 2019;26(12):3203-3211.e5.

42. Neal JT, Li X, Zhu J, et al. Organoid modeling of the tumor immune microenvironment. Cell. 2018;175(7):1972-1988.e16.

43. Chiocca EA, Yu JS, Lukas RV, et al. Regulatable interleukin-12 gene therapy in patients with recurrent high-grade glioma: results of a phase 1 trial. Sci Transl Med. 2019;11(505):eaaw5680.

44. Kleijn A, Kloezeman J, Treffers-Westerlaken E, et al. The in vivo therapeutic efficacy of the oncolytic adenovirus Delta24-RGD is mediated by tumor-specific immunity. PLoS One. 2014;9(5):e97495.

45. Denton NL, Chen CY, Scott TR, Cripe TP. Tumor-associated macrophages in oncolytic virotherapy: friend or foe? Biomedicines. 2016;4(3):E13.

46. Lang FF. DNX-2401 with Interferon Gamma (IFN- $\gamma$ ) for Recurrent Glioblastoma or Gliosarcoma Brain Tumors (TARGET-I). Accessed December 16, 2020. https://clinicaltrials. gov/ct2/show/NCT02197169

47. Tejada S. Virus DNX2401 and Temozolomide in Recurrent Glioblastoma (D24GBM). Accessed December 16, 2020. https://clinicaltrials.gov/ct2/show/NCT01956734

48. Dirven C. Safety Study of Replication-competent Adenovirus (Delta-24-rgd) in Patients with Recurrent Glioblastoma. Accessed December 16, 2020. https://clinicaltrials.gov/ct2/show/ NCT01582516

49. Gallego J. Oncolytic Adenovirus, DNX-2401, for Naive Diffuse Intrinsic Pontine Gliomas. Accessed December 16, 2020. https://clinicaltrials.gov/ct2/show/NCT03178032

50. Zadeh G. Combination Adenovirus + Pembrolizumab to Trigger Immune Virus Effects (CAPTIVE). Accessed December 16, 2020. https://clinicaltrials.gov/ct2/show/NCT02798406

51. Lang FF. Oncolytic Adenovirus DNX-2401 in Treating Patients with Recurrent High-Grade Glioma. Accessed December 16, 2020. https://clinicaltrials.gov/ct2/show/NCT03896568

\section{Disclosures}

Dr. Fueyo: consultant for, shareholder with, and research funding from DNATrix, Inc.; and patent holder for DNX-2401. Dr. Lang: patent holder for DNX-2401.

\section{Author Contributions}

Conception and design: Lang, Ene. Drafting the article: Ene. Reviewed submitted version of manuscript: all authors. Study supervision: Fueyo.

\section{Correspondence}

Frederick F. Lang: The University of Texas MD Anderson Cancer Center, Houston, TX. flang@mdanderson.org. 